\title{
A WMPLS Based Multicast Mechanism in Mobile Ad hoc Network
}

\author{
Li Mengyang \\ Fundamental Education Center of Jilin University, Changchun, China \\ Email: mengyang@jlu.edu.cn \\ Duan Yunna and Zhang Chunfei \\ Computer Science Department in Agriculture division of Jilin University, Changchun, China
}

\begin{abstract}
M erging M PLS into multicast routing protocol in $M$ obile Ad hoc network is an elegant method to enhance the network performance and an efficient solution for multicast scalability and control overhead problems. Based on the W ireless MPLS technology, the mechanism and evaluation of a new multicast protocol, the Label Switching Multicast R outing Protocol (L SM RP) is presented in this paper.
\end{abstract}

Index Terms-WMPLS, multicast, label switching, Mobile Ad hoc network

\section{INTRODUCTION}

An ad hoc network is a network that is dynamically reconfigurable, rapidly deployable, and does not depend on a fixed infrastructure or a central administration. Efficient multicasting in Mobile Ad hoc network faces challenges not encountered in other types of networks such as the mobility of nodes, the tenuous status of communication links, limited resources, and indefinite knowledge of the network topology. In the designing space of multicast protocol, trade-offs must be made between some metrics such as scalability and reliability, flexibility and generality, efficiency and fairness etc. to reach a special objective. On the other hand, the application environments and connection with the Internet require that multicasting should support Quality-of-Service. This is a more challenging requirement because of the inherent characteristics of Mobile Ad hoc Network [1].

MPLS as a QOS provisioning tool has emerged as an elegant solution to meet the bandwidth-management and service requirements for next generation IP based backbone network. We think that multicast and MPLS are two complementary technologies and multicasting over MPLS networks can benefit from the reduction of multicast traffic on one hand, and MPLS' flexibility, speed and quality of service on the other hand. Merging MPLS into multicast routing protocol in Mobile Ad hoc network is not only a viable approach, but an elegant solution to enhance the network performance and an efficient solution for multicast scalability and control overhand problems.

In this paper, we present at first the mechanism of a new multicast protocol, the Label Switching Multicast Routing Protocol (LSMRP) for Mobile Ad hoc network, which is based on Wireless MPLS technology.
Secondly, to enable completely separating of control plane and data plane in the mobile ad hoc network, a framework for MAC layer multicast packet forwarding is proposed. A forwarding node in a multicast group can shift the packet forwarding functionality away from the host processor to the wireless network interface card. This is made possible by carrying a label in RTS/ACK control packet of the 802.11 DCF channel access scheme which allowed the MAC layer to determine the packet's next hop. Using this novel label-driven packet forwarding mechanism within LSMRP multicast architecture can dramatically enhance the performance of the whole networks.

Finally, we conducted an extensive performance evaluation of LSMRP and ODMRP (On-Demand Multicast Routing Protocol) [2] which explores the effectiveness of different multicast mechanisms in a wide range of Mobile Ad hoc network simulation scenarios. The results of simulation show that LSMRP works well and generates less control packet overhead than ODMRP.

\section{LABEL Switching Multicast Routing Mechanism}

To enable MPLS based multicasting, the multicast tree with JOIN (), LEAVE (), DESTROY (), and RPF () messages should be directly implemented in LDP signaling protocol. With new and extended messages introduced to the CD-LDP, LSMRP sets up and maintains multicast forwarding state in an on-demand fashion. The features of LSMRP can be summarized as follows:

- LSMRP is designed to work independently of the unicast protocol used in the Mobile Ad hoc network and can thus work with any unicast protocol or even without a unicast protocol.

- LSMRP is completely distributed and does not rely on any centralized coordination or control.

- LSMRP adapts its behavior to various network conditions and different sending pattern, can automatically switch to appropriate forwarding topology (tree, mesh, and network-wide flooding) to enable efficient multicasting.

- LSMRP uses no periodic control packet, periodic neighbor sensing, or periodic routing table exchanges through network-wide floods. 
- LSMRP uses label-driven packet forwarding mechanism to separate control plane and data plane in the Mobil Ad hoc network.

All the features above are derived from the flexibility of label-driven packet forwarding mechanism in MPLS.

\section{A. Multicast Extension to CR-LDP protocol}

LSMRP uses CR-LDP signaling protocol to support multicast operations such as forwarding topology setting up and maintenance. Due to the lack of multicast mechanism in CR-LDP (RFC 3212), it's necessary to introduce new objects into signaling messages within CRLDP $[3,5,6]$.

Objects in CR-LDP are encoded into TLVs (type, length, and value) so as to make the protocol extendable. In LSMRP, there are a few of TLVs $[10,13]$ are modified to support necessary multicast operations, which include multicast Forwarding Equivalence Classes (FEC) and extended Notification Message used to carry the control packets.

\begin{tabular}{|c|c|c|c|}
\hline 0 & 0 & Multicast FEC & Length \\
\hline V & & Reserved & Multicast ID \\
\hline \multicolumn{4}{|c|}{ Multicasting IP Source Address } \\
\hline \multicolumn{4}{|c|}{ Multicasting IP Group Address } \\
\hline
\end{tabular}

In an ingress router, FEC is assigned to every packet which enters into a WMPLS network, and then is encoded into a fixed-length value according to relative information carried by packet, and thus the Label is generated. Ingress router can use various-level information (network layer, transport layer, and even application layer) contained in a packet to assign FECs to obtain various objectives.

LSMRP has defined a new kind of FEC called Multicast FEC, which is illustrated by Fig. 1. The Multicast FEC is encoded using multicast group address and IP address of the multicast source node, then multicast packets with the same group address or source address are assigned to the same FEC.

When multicasting in a WMPLS network begin, LSMRP takes the sender wireless node as an ingress router, and every node in multicast forwarding mesh will allocate a local Label to each incoming packet according to its FEC. Then, multicast forwarding will be driven by the Label. [7, 8, 11]

Assigning FEC to multicast packets is crucial to the performance of LSMRP. The assigning method is flexible in order to meet various demands in a specific application, and this mechanism can also provide solutions to implement QoS Routing and Flow Engineering in wireless ad hoc network. [9, 12, 15]

\section{B. Messages in LSM RP}

LSMRP uses ten types of message to implement communication among multicast nodes, and to support all multicast operations. All of the messages are formatted based on standard CR-LDP signaling protocol. [10]

\section{Label-Request Message}

This message is used by multicast source node to notify all of its multicast members of the FEC which it has assigned. And the message will be sent out either by network flooding when a multicast tree is setup at first time, or by unicast via reverse path route (RPF) when reply the Local-Repair-REQ message during a local repair process.

\section{Label-Mapping Message}

This message is sent out by multicast receiver and forwarder to allocate local label according to the known FEC and establish the multicast label switching path (LSP).

\section{Label-Mapping RPLY Message}

This message is used by source node to confirm the receiver's acknowledgment of FEC, and will be sent out by the source node via RPF.

\section{JOIN-REQ Message}

This message is used by multicast receiver either to establish a new multicast tree or to notify the source node of the multicast forwarding mash failure. The message will be sent out in three cases: when the receiver will setup multicast tree at first time, when waiting for LabelMapping-RPLY message is timeout, and when a global mesh repair process is initiated.

\section{Local-Repair-REQ Message}

This message is used to notify the source node of the upstream link failure, and will be sent out by the node which firstly detects link failure.

6. Local-Repair-Notification Message

This message is used to notify the downstream nodes that a local repair process has already begun, and thus can prevent repeated repair processes initiated by downstream nodes which have detected the upstream link failure.

7. Global-Repair-REQ Message

This message is used by multicast receiver to notify the source node that a global repair process will be initiated. The message is sent out through network flooding when receiver's waiting time for Label-RPLY message exceeds threshold.

8. Keep-Alive Message

This message is used by multicast source node to keep alive the forwarding mesh for a period of time when there are no packets to send out temporarily.

9. Label-Release Message

This message is used by multicast source node to release an assigned FEC throughout the whole forwarding mesh, and is sent out whenever a multicast session is over.

10. Label-Withdraw Message

This message is sent out by multicast receiver through broadcast with one hop count to notify upstream nodes of its leave from the multicast group.

\section{D ata Structure}

LSMRP uses following data structures stored in every wireless node to support all multicasting operations:

1. Forwarding Equivalence Classes (FEC) table, $\{$ FEC, actor, local label, packet interval time, time factor, keep alive count, pointer to output Label\}, which is used to manage FEC information and maintain multicasting topology. FEC table is illustrated in Fig. 2. 


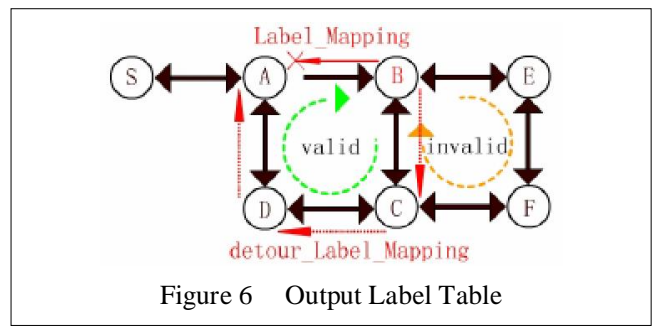

When node B sends Label-Mapping message to node $A$ via $R P F$, the unidirectional link A $B$ can be detected with the help of ACK handshaking and often also RTS/CTS handshaking in the IEEE 802.11 DCF MAC. Then node $\mathrm{B}$ initiates a detour-path finding procedure in order to send Label-Mapping message to node A via this detour-path.

In LSMRP, finding a path means flagging all the nodes along the path as forwarding nodes. Upon detecting a unidirectional link, LSMRP will discover a detour loop and will flag nodes that are part of the path from node B to node $\mathrm{A}$ in the loop. To start, the Label-Request message is flooded from $\mathrm{B}$ into the network. The flood reaches node $A$ through the path $\mathrm{B} \ddagger \mathrm{C} \neq \mathrm{D} \neq \mathrm{A}$. Upon receiving the Label-Request message, node $A$ sends back a Label-Mapping message using the reverse path. The detour loop discovery procedure will find the loop

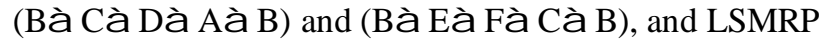
accepts only loops in which one node is closer to source than the initiator itself. This guarantees convergence.

\section{F. Adapting to Varying Network Environments}

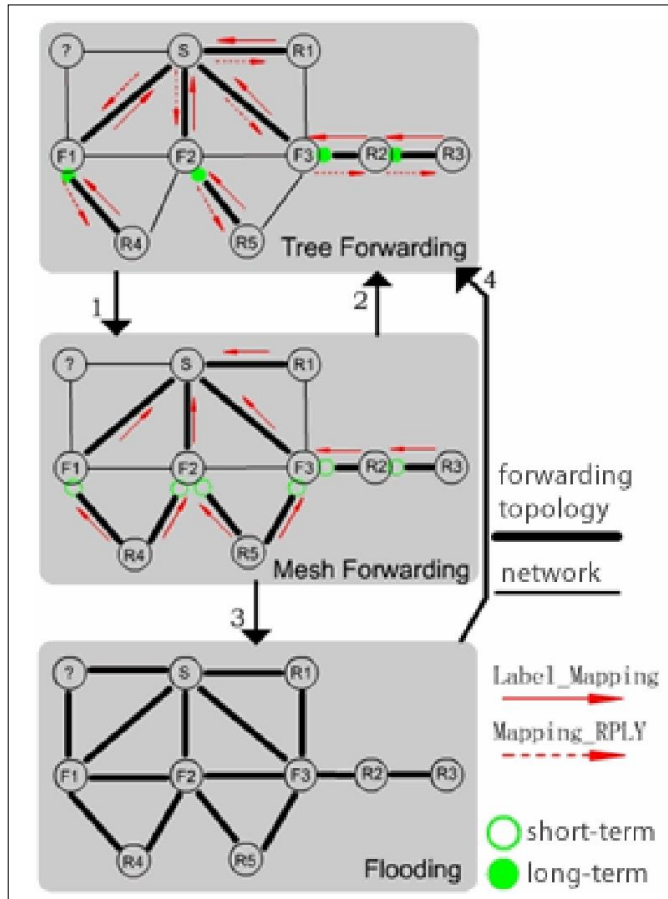

Figure 7 Multicast topology patterns

Forwarding topology is the key element to affect the performance of Mobile Ad hoc Network, and it should have the ability to adapt to changing environment.
LSMRP uses three different kinds of topology which can be dynamically shifted to appropriately match the varying network conditions and different kinds of application modes. They are: multicasting tree, multicasting mesh, and flooding.

Fig. 7 shows how these three kinds of topology shift during a multicast session.

- tree to mesh

After the first round of setup, packets are forwarded via a multicast tree. Whenever a receiver does not receive Label-Mapping RPLY message within a given time slot, it will flood the Label-Mapping message to launch the second round setup process. Then, each neighbor node will set a temporary output label for the receiver, and packets will be forwarded via a mesh during a period of time.

- mesh to tree

When source node receive Label-Mapping message via flooding, it will echo with a LabelMapping RPLY message via an optimal inverse path to the destination. Nodes on this path will set an output label for destination node with a period of time longer than that of temporary label. Then, the forwarding topology shifts to a tree again.

- mesh to flooding

To handle with link failure problem in Mobile Ad hoc network, forwarding nodes will frequently launch the local repair process. Whenever source node finds there are too much repairing, it can be determine that the network topology is under too much frequent fluctuation. Then, source node will take steps to send packets via flooding by which label switching is not used.

- flooding to tree

When receiver senses the flooding packets, it will launch the next stage setup process, and a new multicasting tree will be formed to match the new network environment.

\section{MAC LAYER PACKET FORWARDING THROUTH LABEL SWITCHING}

LSMRP uses label-switching mechanism in network layer to maintain multicast topology effectively. In order to get better network performance, there is a need to separate the packet forwarding from network layer to MAC layer, and implement MAC layer forwarding through label switching [3]. This demands MAC layer has the ability to identify the next hop of packets. LSMRP transfers Label-switching Forwarding table to MAC layer synchronically, so that MAC layer can determine the next hop of packets without any operation in network layer [4].

The ACK mechanism in MAC layer is crucial for LSMRP to sense changes of network topology [5]. Therefore, we use IEEE 802.11 DCF as the media access control protocol and extend it to support label-switching 
in MAC layer. Fig. 8 and Fig. 9 depicts the extending messages and time slots used by LSMRP.

When network-layer forwarding works in mesh and flooding pattern, MAC layer will broadcast to all neighboring nodes, which will in turn receive all broadcasting frames and determine whether to forward them. Broadcasting will waste processing resources of some irrelevant nodes [6]. To solve this problem, LSMRP uses Wireless MPLS packet format to carry data-load and implement constrained broadcasting. In the header of WMPLS packet, the label stack can carry multiple output labels on demand. A convergence node in multicast topology sends packets in this multi-label fashion, which can be used by neighboring nodes to determine whether to forward or just to drop these packets.

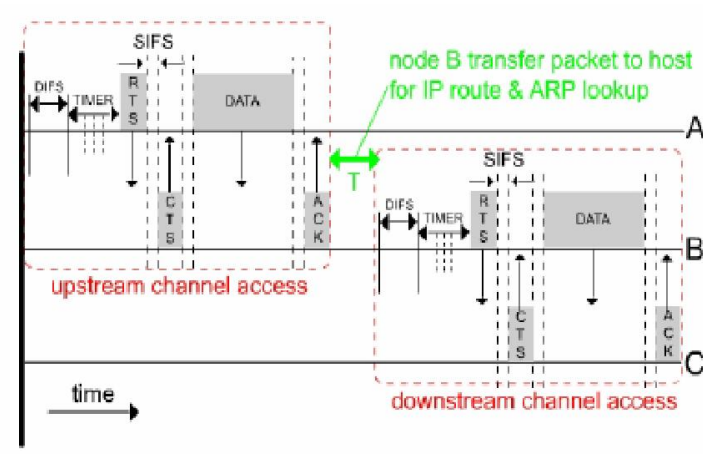

Figure 8 Packet forwarding in MAC layer through labelswitching

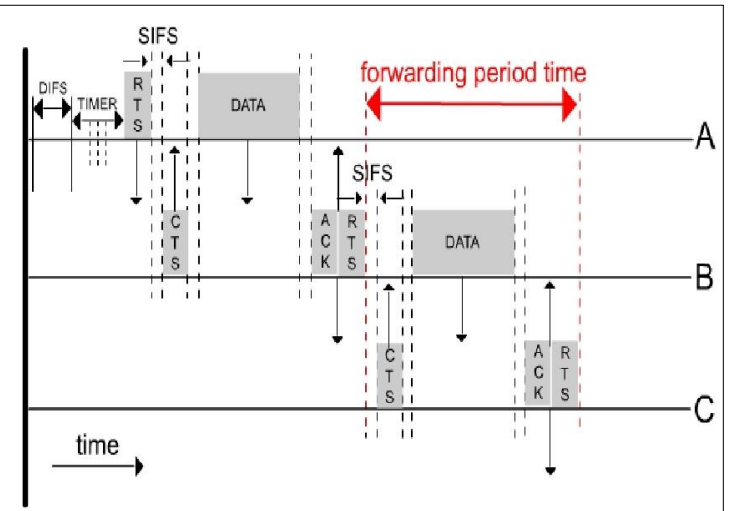

Figure 9 Packet forwarding in MAC layer through labelswitching

\section{SIMULATION RESULTS}

We use Network Simulator 2.1b8 with CMU wireless extensions to evaluate the performance of LSMRP. And we choose the On Demand Multicast Routing Protocol (ODMRP) as a comparison.

\section{A. Network and Communication Model}

The minimum degree of node $V$ in the network is $d$, and the minimum number of common neighbors shared by two neighbors $\mathrm{U}$ and $\mathrm{V}$ is $\mathrm{d}_{0}$. Accidental collisions and interference are possible, but deliberate collisions by faulty nodes are not allowed. Address-spoofing is also assumed not to occur, i.e. for each message, the node that put that message on the air is uniquely and correctly identifiable. If a node transmits a message, the probability that a neighbor successfully receivers it is $p_{s}$. Thus possible errors due to fading, interference etc. are subsumed in the error probability $\left(1-p_{s}\right)$. We define a timeout $T$ and a probability $p_{a}$ such that if a packet was put into a node's outgoing queue at time $t$, then with probability at least $p_{a}$, it gets a chance to transmit it by timet $+T$. The choice of $T$ is such that $p_{a}$ may be large It may be obtained via loose estimates on network density (and hence contention). All nodes possess a single transceiver and operate on a single channel. They also use a single transmission rate, and all valid messages are of a predetermined (and equal) size.

We assume that the minimum time between two successive packet transmissions is $\mathrm{t}_{\delta}$. Note that $\mathrm{t}_{\delta}$ can be no less than the transmission time of a packet, the maximum propagation delay is $\mathrm{d}_{\text {prop }} \max$. Nodes are externally synchronized with bound $\mathrm{D}<\frac{1}{2}\left(\mathrm{t}_{\delta}-\mathrm{d}_{\text {prop }} \max \right)$. Such high-precision synchronization may be feasible in the near future with the advent of on-chip atomic clocks. Also observe that given the maximum clock skew $D$ in the network, it is possible to ensure that the condition holds by padding all messages with extra bits to increase the transmission time so that $\mathrm{t}_{\delta}>2 \mathrm{D}+\mathrm{d}_{\text {prob }}{ }^{\max }$, and the required condition holds. This condition ensures that if a node sends out two different messages on the same channel, then the nodelocal time at which any non-faulty node receives the later message shall always be greater than the node-local time at which any non-faulty node receives the first message.

Distinct messages sent by a particular source are distinguished via identifiers that we shall denote as id. The id is a number in some range $[0, M A X]$. Individual nodes choose the sequence of ids for their messages in some privately determined pseudo-random manner (such that ids are re-used only after large intervals of time). Thus, if a node sends two conflicting versions of the same message, it implies that they both have the same id, but different values. Messages are represented as $\mathrm{m}$ (src, id, value). We assume that value can take values 0 or 1 .

\section{B. Simulation System M odel}

We consider an ad hoc network consisting of $\mathrm{N}$ mobile nodes, having a uniform stationary distribution over a square area, of dimension $b \times b$. The multi-access scheme is direct-sequence CDMA (DS-CDMA) and three types of receivers are considered: the matched filter (MF), the decorrelator, and the linear minimum men squared 
error receiver (MMSE). All nodes use independent, randomly generated and normalized spreading sequences of length $L$. For simplicity, we assume that all nodes transmit with the same power, $P_{t}$, and we define the signal-to-noise ratio (SNR), as the ratio between the transmitted power and the noise power: $\mathrm{SNR}=\mathrm{P}_{\mathrm{t}} / \sigma^{2}$. As in [11], we consider a transmitter oriented protocol, in which each transmitting node has its own signature sequence. Although this implementation yields more complex receivers and longer acquisition times, it has very good capturing probabilities, allowing multiple packet reception at the same receiver node. To avoid collisions, multiple concurrent transmissions from the same node are not allowed; instead transmissions from one node to multiple destination nodes are time multiplexed. We assume that all nodes are active at a given time (to transmit their own packets or relayed traffic), although the analysis can be easily extended to the case in which only a fraction $\beta$ of nodes are active (in which case interference is reduced by a factor of $1 / \beta$ ).

The path loss model is usually characterized by three zones: the near field zone, the free space path loss zone and the excess path loss zone. The near field zone extends to a distance of

$$
\mathrm{d}_{1}=\frac{2 \mathrm{D}_{\max }^{2}}{\lambda}
$$

Where $D_{\max }$ is the largest dimension of the antenna, and $\lambda$ is the wavelength of the carrier. The signal attenuation in this zone is the highest and it is usually not modeled for typical applications. For an antenna dimension of $D_{\max }=\lambda / 2$, the near-field zone extends to $d_{1}=\lambda / 2$. In this paper we approximate the path loss model, and we assume that no reception is possible within distance $d<d_{m}$, where $d_{m}=\lambda>d_{1}$.

$$
\text { For distances } \quad d \geq d_{m}
$$

and $d \leq d_{2}=\frac{4 h_{t} h_{r}}{\lambda}\left(h_{t}\right.$ and $h_{r}$ are the heights of the transmitter and receiver antennas, respectively), the free space propagation model applies. For antenna heights greater or equal to 1 meter (a reasonable value for ad hoc networks), and $3 \mathrm{GHz}$ frequency ( $\lambda=0.1$ meters), we have $d_{2} \geq 40$ meters. Thus, since we are considering low range transmissions in the ad hoc networks, in our analysis we consider a free space propagation path loss model for which the received power is given as:

$$
P_{r}=P_{t}^{*} G_{t} G_{r} \frac{\lambda^{2}}{(4 \pi d)^{2}}=P_{t} \frac{\lambda^{2}}{d^{2}}=P_{t} h
$$

where $P_{t}$ represents the above-defined transmitted power, which incorporates also the transmitting and receiving antenna gains and the constant $1 /(4 \pi)^{2}$, and $h=\lambda^{2} / d^{2}$ is the link gain.

The traffic can be directly transmitted between any two nodes, or it can be relayed through intermediate nodes. It is assumed that the end-to-end delay can be measured in the number of hops required for a route to be completed. The QoS requirements for the ad hoc network are the bit error rate (mapped into a signal-to-interference ratio requirement: SIR), the average source-destination throughput ( $\left.T_{S-D}\right)$, and the transmission delay. Both the throughput and the delay are influenced by the maximum number of hops allowed for a connection and consequently, by the network diameter $D$. Using arguments similar to those in [14], a simplified computation shows that, if the number of hops for a transmission is $D$, then each node generates $\mathrm{DI}(\mathrm{N})$ traffic for other nodes, where $(\mathrm{N})$ represents the traffic generation rate for a given node. Thus, the total traffic in the network must meet the stability condition $D I(N) N \leq N W / L$, whereW is the system bandwidth. This implies that the average source-destination throughput that can be supported by the network must meet the condition

$$
\mathrm{T}_{\mathrm{S}-\mathrm{D}} \leq \frac{\mathrm{W}}{\mathrm{LD}}
$$

We

$$
\text { note that }
$$
throughput $\quad T_{S-D}(N)=I(N) \leq W / L D(N)$ is actually dependent on the number of nodes in the network $\mathrm{N}$, which influences the achievable network diameter.

\section{B. Simulation Enironment}

- Wireless model: Lucent Wave LAN 802.11, 2Mbps.

- Mobile scenario: Random Way Point Model, 100 nodes within $1000 * 800 \mathrm{~m}^{2}$, and 300 nodes within $2200 * 1260 \mathrm{~m}^{2} ; 2 \mathrm{~m} / \mathrm{s}$ motion velocity.

- Communication scenario: multicast sender and receiver join group randomly; multiple groups is allowed with maximum 10 senders and 50 receivers per group; the packet flow type is CBR.

\section{Metrics}

- Packet Delivery Ratio (PDR) measures ratio of successfully received packets within a group and all sent packets by multicast sender.

- End-to-End Delay (EED) measures the average delay from sender to receivers in a group.

- Control Packet Load (CPL) records the numbers of all control packets in a multicast session. 


\section{Results}

- Affection of various number of receiver

TABLE I. AFFECTION OF VARIOUS NUMBER OF RECEIVER

\begin{tabular}{|l|c|c|c|c|}
\hline \multirow{2}{*}{ metrics } & protocol & $(1,1,10)$ & $(1,1,30)$ & $(2,5,50)$ \\
\hline \multirow{2}{*}{ EED } & LSMRP & 16 & 18 & 31 \\
\cline { 2 - 5 } & ODMRP & 17 & 20 & 25 \\
\hline \multirow{2}{*}{ CPL } & LSMRP & 0.1 & 0.1 & 0.5 \\
\cline { 2 - 5 } & ODMRP & 0.4 & 0.6 & 1.3 \\
\hline \multirow{2}{*}{ PDR } & LSMRP & 0.99 & 0.99 & 0.99 \\
\cline { 2 - 5 } & ODMRP & 0.99 & 0.99 & 0.99 \\
\hline
\end{tabular}

An increase of the number of receiver leads to more forwarding nodes and packets, so that results in higher PDR in both LSMRP and ODMRP. But ODMRP generates more multicast forwarding nodes than LSMRP. TABLE I shows metrics of two protocols respectively. And we use "(group number, sender number, receiver number)" to represent a multicasting communication scenario.

- Affection of various number of sender

When the number of sender increases, LSMRP remains a relatively high PDR, while ODMRP exhibits a slow decrease of PDR. The reason of these phenomena is that more senders will generate more flooding packets in ODMRP, and then the packet crashing ratio became higher and higher, eventually leads to large number of dropped packets.

TABLE II. AFFECTION OF VARIOUS NUMBER OF SENDER

\begin{tabular}{|l|c|c|c|c|}
\hline metrics & protocol & $(1,1,10)$ & $(1,1,30)$ & $(2,5,50)$ \\
\hline \multirow{2}{*}{ EED } & LSMRP & 12 & 20 & 23 \\
\cline { 2 - 5 } & ODMRP & 11 & 15 & 20 \\
\hline \multirow{2}{*}{ CPL } & LSMRP & 0.16 & 0.35 & 0.36 \\
\cline { 2 - 5 } & ODMRP & 0.65 & 3.1 & 6.3 \\
\hline \multirow{2}{*}{ PDR } & LSMRP & 0.98 & 0.98 & 0.97 \\
\cline { 2 - 5 } & ODMRP & 0.99 & 0.99 & 0.93 \\
\hline
\end{tabular}

\section{CONCLUSION}

Our study proved that using MPLS with multicast in the Mobile Ad hoc network has many benefits not only for reducing multicast forwarding states but also for traffic engineering and QoS issues. Merging MPLS into multicast routing protocol in Mobile Ad hoc network will give rise to more potential advantages, such as saving more processing resources and energy of mobile host, timely adapting to the network conditions with protocol's behavior, and extending wired network services to wireless network more conveniently, etc.

\section{REFERENCES}

[1] Garbinato B , Rupp. P. From ad hoc networks to ad hoc applications. Telecommunications, 2003. ConTEL, 2003. Proceedings of the $7^{\text {th }}$ International Conference on , Vol. 1 , June 11 13, 2003 pp. 145 149

[2] Sang Ho Bae, , Sung Ju Lee, William Su , and Mario Gerla. The design, implementation, and performance evaluation of the on-demand multicast routing protocol in multi-hop wireless networks. IEEE Network January/February 2000 , pp. 70-77.

[3] Ranveer Chandra, Paramvir Bahl, and Pradeep Bahl, "Multinet: Connecting to multipleIEEE 802.11 networks using a single wireless card ," in IEEE Infocom , Hong Kong, March 2004.

[4] A. El-Gamal , J. Mammen , B. Prabhakar , and D. Shah. Throughput-delay trade-offs in wireless networks. In Proc. of IEEE Infocom, March 2004.

[5] S. Xu and T. Saadawi. "Does the IEEE 802.11 MAC Protocol Work Well in Multi-hop Wireless Ad Hoc Networks?" , IEEE Communications Magazine. 39(6) , June 2001 , pp. 130-137.

[6] Paramvir Bahl , Ranveer Chandra, and John Dunagan, "Ssch: Slotted seeded channel hopping for capacity improvement in ieee 802.11 ad-hoc wireless networks ," in ACM M obicom , 2004.

[7] N.Gupta and S.R.Das. A capacity and utilization study of mobile ad hoc networks. In proceedings of the $26^{\text {th }}$ Annual Conference on Local Computer Networks, pages 576-583, November 2001.

[8] C.-Y. Koo, "Broadcast in radio networks tolerating byzantine adversarial behavior," in Proceedings of the twenty-third annual ACM symposium on Principles of distributed computing. ACM Press, 2004, pp. 275-282.

[9] V. Bhandari and N. H. Vaidya, "On reliable broadcast in a radio network," Technical Report, CSL, UIUC, May 2005.

[10] A. Pelc and D. Peleg, "Broadcasting with locally bounded byzantine faults," Information Processing Letters, vol. 93, no. 3, pp. 109-115, Feb 2005.

[11] V. Bhandari and N. H. Vaidya, "On reliable broadcast in a radio network," in Proceedings of the 24th annual ACM symposium on Principles of distributed computing, 2005.

[12] E. M. Belding-Royer and C. E. Perkins. Evolution and Future Directions of the Ad hoc On-Demand Distance Vector Routing Protocol. Ad hoc Networks Journal, 1(1), July 2003.

[13] S. Knappe, L. Liew, V. Shah, P. Schwindt, J. Moreland, L. Hollberg, and J. Kitching, "A microfabricated atomic clock,” Appl. Phys. Lett., vol. 85, 2004.

[14] S. Nesargi and R. Prakash. MANETconf: Configuration of Hosts in a Mobile Ad Hoc Network. In Proceedings of IEEE Infocom 2002, New York, USA, June 2002.

[15] K. Weniger. Passive Duplicate Address Detection in Mobile Ad Hoc Networks. In WCNC, Florence, Italy, February 2003.

Li mengyang (male, 1972- ), associate professor at Jilin University in Changchun of China.

Educational background: 1. Information Engineering University, Zhengzhou, China, majored in cryptography (19891993). 2. computer science and technology dept. , Jilin University, Jilin, China, majored in computer system and architecture (2002-2005).

Research interests: 1 . Course design of "applied technology of bioinformatics towards junior undergraduates". 2. Wireless ad hoc network architecture and its application to web-learning, and performance evaluation of wireless sensor network. 3 . Biological software architecture and development automation, application study of computational intelligence in structural bioinformatics, and protein interaction predictions from diverse sources. 